\title{
The Moderating Role of Age in Responses to Direct-to-Consumer Prescription Drug Advertising
}

\begin{abstract}
Age is an important factor that can influence processing of and response to health messages. Many studies examining evaluations of and responses to direct-to-consumer prescription drug advertising (DTCA) have incorporated age as a predictor variable, moderating variable, or sample criterion. However, findings have been inconsistent. This study attempts to add clarity to this body of research by assessing age differences in the antecedent factors of various DTCA outcomes. A multigroup structural equation modeling analysis revealed several significant differences in variable relationships between older (50+) and younger $(<50)$ adults. Overall, older adults exhibited greater complexity in their consideration of DTCA than younger adults in terms of the sheer number of significant relationships within the model. In particular, trust in mediated health information sources and trust in one's physician appear to be more relevant predictors for older adults. Trust in DTCA was also distinguished as having an inverse relationship with behavioral intentions among older adults while showing a straightforward positive association with attention among younger adults. Further analysis indicated health status accounted for some but not all of the age differences. It is suggested that younger adults are more open to seeking additional information following DTCA exposure while older adults remain ambivalent.
\end{abstract}

Keywords: direct-to-consumer; prescription drug; advertising; age differences; structural equation model; trust 
Communication scholars have long recognized the importance of individual differences in determining communication outcomes. Characteristics related to personality (e.g., need for cognition [Cacioppo \& Petty, 1982]), motivation (e.g., involvement [Petty, Cacioppo, \& Schumann, 1983]), and demographics are frequently examined as relevant factors moderating responses to messages, health-related and otherwise. Among these various characteristics, the factor of age is often underappreciated and therefore understudied. Within health communication, this gap is particularly stark in the examination of mass media campaigns. Recently, Southwell (2010) argued for instituting a "life-span approach" to health campaign evaluation that acknowledges fundamental differences in information processing needs and abilities between age groups. For example, changes in brain plasticity can reduce learning and memory among the elderly and impact the process by which different age segments respond to persuasive health messages. Thus, it was argued that examination of age as a moderator in models of health campaign effectiveness is crucial. One specific category of health messages to which this life-span approach can and should be extended is direct-to-consumer prescription drug advertising (DTCA).

DTCA represents a form of health communication intended to inform consumers about health conditions and prescription treatments of those conditions with the ultimate goal of motivating consumers to consult their physicians about the advertised medication. Age is likely to be a critical factor in assessing response to these ads for a number of key reasons. For one, older consumers are targeted by DTCA more often because they tend to experience more health issues and have a higher rate of medication usage (Ball, Manika, \& Stout, 2011; DeLorme, Huh, \& Reid, 2007). This implies that, on the whole, relevance of DTCA varies by age. However, evidence has demonstrated DTCA content to be complex and difficult to comprehend 
(Kaphingst, Rudd, DeJong, \& Daltroy, 2005). This complexity is incompatible with the documented decline in cognitive abilities among seniors (Craik \& McDowd, 1987). Hence, increased relevance does not ensure adequate processing and evaluation of DTCA. Relatedly, elderly individuals are considered a vulnerable population that is more susceptible to misleading ad claims. Given prevalent concerns regarding the biased promotional slant and questionable informational value of prescription drug ads (Royne \& Myers, 2008), it is of interest to understand how determinations of DTCA trustworthiness differ by age.

Other scholars have likewise recognized the worthiness of examining the role of age in DTCA effectiveness, but the treatment of age as a defining criteria of the study sample (e.g., An \& Mituri, 2011; DeLorme, Huh, \& Reid, 2007), a control or independent variable in regression analysis (e.g., Hausman, 2008; Krezmien, Wanzer, Servoss, \& LaBelle, 2011), or a primary factor for comparing DTCA perceptions and responses across groups (e.g., Ball, Manika, \& Stout, 2011; DeLorme, Huh, \& Reid, 2006) has varied. It is difficult to draw overarching conclusions from this body of research since diversity in the way age is examined have produced inconsistent results. Additional investigation is warranted.

The current analysis applies age as a moderating factor in testing a model of DTCA evaluation and response across older and younger segments of the population. This analysis allows for an assessment of the robustness of the model and builds upon previous age comparison research in the DTCA literature. In particular, the study reported here examines how the relationships between a wide range of variables differ between older and younger consumers. In addition, while previous DTCA research has typically approached age comparisons with exploratory research questions, this paper adds a bit more rigor by presenting theory-based predictions of age differences among variable relationships. 
This paper provides an overview of age differences in the DTCA literature followed by a brief review of theoretical approaches to explaining age difference patterns. This combination of empirical evidence and theory offer a foundation for interpreting the significant differences we found between older and younger adults, as discussed in the final section.

\section{LITERATURE REVIEW}

\section{Age in DTCA Research}

A review of the DTCA literature revealed age to be one of the most common individual characteristics incorporated into the study design (see Table 1 for a summary). The majority of these studies included age as a control or predictor variable. However, the apparent role of age in DTCA responses has been decidedly mixed. In regards to behavioral responses, several studies concluded age was a not a differentiating factor (Ball, Manika, \& Stout, 2011; DeLorme, Huh, \& Reid, 2011; Huh, DeLorme, \& Reid, 2005; Lee, Salmon, \& Paek, 2007; Peyrot, Alperstein, Van Doren, \& Poli, 1998). When a significant difference was present, older consumers were more likely to ask a health professional about a prescription drug (Choi \& Lee, 2007; DeLorme, Huh, \& Reid, 2006; Krezmien et al., 2011; Mehta \& Purvis, 2003) but less willing to request a prescription for that drug compared to younger consumers (Choi \& Lee, 2007; Datti \& Carter, 2006; Hausman, 2008; Mehta \& Purvis, 2003).

\footnotetext{
Place table 1 about here
}

Age differences in perceptions of DTCA have also varied. If one looks for common themes, seniors have more often exhibited lower overall attitudes toward DTCA (Lee, Salmon, \& Paek, 2007; Yuan, 2008) and appeared more critical of the ads' perceived informativeness and trustworthiness (An \& Mituri, 2011; Ball, Manika, \& Stout, 2011; DeLorme, Huh, \& Reid, 2007; Foley, 2001; Grenard, Uy, Pagán, \& Frosch, 2011). However, evidence has suggested age is 
positively associated with greater perceived utility of DTCA (Huh, DeLorme, \& Reid, 2004), perhaps especially for raising awareness of particular prescription drugs (DeLorme, Huh, \& Reid, 2007; Grenard et al., 2011). Older consumers also rated prescription drug messages as easy to understand (An \& Mituri, 2011) but learned less from the ads than younger individuals (Abernathy \& Adams-Price, 2006).

In summary, age has not always been a differentiating factor in study results. When differences are found, older consumers seem to find the ads more useful and are at times more willing to at least inquire about a drug with a health professional, but their overall evaluations of DTCA are less likely to be favorable. In comparison, younger consumers demonstrate greater acceptance of DTCA and consider the ads more informative. However, this pattern does not hold consistently across studies. A major limitation is that analyses have typically failed to fully examine the nature of the relationships between different predictor and outcome variables across age groups. Understanding the extent to which trust, attitudes, and behaviors are differentially related to one another and based on different antecedents by age might shed light on current inconsistencies. A small number of initial exploratory studies suggest older and younger segments may base DTCA perceptions and responses on different factors (Ball, Manika, \& Stout, 2011; DeLorme, Huh, \& Reid, 2006; Huh, DeLorme, \& Reid, 2004), but more thorough and theoretically-based investigation is needed.

\section{Theoretical Perspectives on Age}

The literature shows a consistent pattern of reductions in memory, speed and depth of information processing, and ability to integrate new information with existing stored knowledge as people age (Brown \& Park, 2003; Craik \& McDowd, 1987). Elderly adults also tend to be more trusting (Castle et al., 2012; Li \& Fung, 2013) while at the same time more risk averse than 
younger counterparts (Simcock, Sudbury, \& Wright, 2006). There are different theoretical accounts that explain these findings. The cognitive approach ascribes deterioration of cognitive functions to neurological decay and declining mental resources. In particular, ability to engage in controlled, effortful processing (e.g., recalling specific details from memory) is impaired while automatic processing (e.g., recognition) remains intact (Craik \& Jennings, 1992). In addition, the theory of disinhibition suggests seniors' decreased ability to inhibit attention to distractions such as irrelevant information leads to slower and less focused information processing (Hasher, Zacks, \& May, 1999). To compensate for these deficits, older adults adopt a more heuristic style of information processing relying on available cues and more general accumulated knowledge (Brown \& Park, 2003).

Socioemotional selectivity theory offers an affective underpinning for observed age differences by asserting that goals guiding behavior differ based on one's perceived time horizon (Carstensen, 1992; Carstensen, Isaacsowitz, \& Charles, 1999). When a person perceives a lengthy future, expansive or knowledge-related goals stimulate knowledge acquisition through the pursuit of information and novel experiences. If instead time is perceived as limited, emotion regulation or "emotionally meaningful" goals shift focus to achieving positive feeling states and avoiding negative ones through familiar experiences and close relationships. In-depth seeking and processing of information is not considered necessary under this circumstance. While time perspective is independent of age, older people are more likely to sense their future as limited compared to younger adults (Carstensen, Isaacsowitz, \& Charles, 1999).

These two theoretical perspectives are not mutually exclusive. Cognitive theory involves ability while socioemotional selectivity theory involves motivation. Since message processing and judgment are affected by both ability and motivation, these two approaches provide a well- 
rounded view supporting empirical evidence for age differences and the need to understand how these complex differences are manifested in response to health messages such as DTCA.

\section{PROPOSED MODEL FRAMEWORK}

The objective of the current analysis was to test a model of DTCA perceptions and responses across two age groups to determine if variable relationships differed in strength, significance, and/or direction by age. The model was initially proposed by the authors and validated on a general population sample (CITATION OMITTED FOR BLIND REVIEW). At the heart of the model is the concept of DTCA trust. In accordance with trust scholarship, DTCA trust is defined as the confident belief that prescription drug advertising has been created with competence, benevolence, and integrity.

The construction of the model was based on the premise that DTCA trust would act as a mediator between various predictor variables and key outcome variables. Based on inferential theory, individuals infer the trustworthiness of communication from characteristics of the message, the source, and the broader context (Keren 2007; Malaviya, Kisielius, and Sternthal 1996). Based on empirical findings from the DTCA literature, these three categories of trust cues were applied as perceptions of the accuracy and value of information in prescription drug ads (message); perceived credibility of pharmaceutical corporations (source); and psychographic variables including trust toward one's physician, trust toward mediated health information sources, and attitude toward advertising in general (context). Consistent with previous theoretical and empirical work on DTCA effectiveness (e.g., Menon, Deshpande, Zinkhan, \& Perri, 2004) and trust (e.g., McKnight and Chervany 2001-2002; Soh, Reid, and King 2009), the selected outcome variables were attention to DTCA, attitudes toward DTCA, and behavioral intentions (see Figure 1). 
Place figure 1 about here

\section{Predicted Differences by Age}

Theory and empirical evidence allow for some general predictions regarding age differences in the model relationships. In particular, cognitive and socioemotional selectivity theories indicate tendencies toward deeper information processing decline with age. Evidence supports the implication that older adults are more likely to process information heuristically even when the information is relevant (Brown \& Park, 2002; Gaeth \& Heath, 1987). In addition, consistent with the postulate of socioemotional selectivity theory that a shorter time horizon increases desire to avoid negative experiences, older adults demonstrate a stronger averseness to risk and place more emphasis on the trustworthiness of a source (Simcock, Sudbury, \& Wright, 2006).

Hence, it can be expected that attention to DTCA—conceptualized as the allocation of cognitive resources to processing information —will be a more influential determinant of DTCA behavioral responses and DTCA attitudes among younger consumers than older consumers due to differing information processing strategies. Similarly, perceived accuracy and value of DTCA messages (DTCA message perceptions) are likely to be important to both age groups but are expected to be a more dominant factor in the outcomes for younger adults since this perception represents a more specific level of DTCA evaluation. Conversely, older adults' greater reliance on general accumulated knowledge and contextual cues (Brown \& Park, 2003) suggests their behavior will instead be guided more by global attitudes toward DTCA and broader considerations such as trust in mediated health information sources and general attitude toward advertising. Seniors' greater concern with trust and source reputation also implies perceived pharmaceutical corporate credibility and DTCA trust will be stronger predictors of the outcome 
variables for older adults than the younger group. In addition, older adults have expressed greater deference to healthcare professionals and place more trust in physicians than alternative sources of health information compared to younger adults (Menon et al., 2004). Therefore, it is anticipated older adults will show stronger and more negative relationships between trust in one's physician and ad outcomes compared to younger adults.

\section{METHODOLOGY}

\section{Sample}

Members of a web-based, opt-in U.S. online survey panel were invited via email to participate in the survey in exchange for entry into a drawing to win cash prizes. A total of 1036 panel members completed the survey. Participants were divided into younger (18-49 years old) and older adults (50 years old and above) based on trends in consumer research (Simcock, Sudbury, \& Wright, 2006) and common cultural definitions (e.g., AARP eligibility) designating age 50 as entry into senior citizenship. The size of each group was approximately balanced between younger $(n=543)$ and older $(n=493)$ adults. The groups were similar in that a majority were Anglo American, had at least some college education, and had a median income of $\$ 50,000-\$ 75,000$. However, there were significantly more females among the younger adults while the older adults group had a more even male to female ratio. In addition, younger adults were significantly less likely to report past six month exposure to prescription drug ads than older adults. See Table 2 for full sample descriptives.

Place table 2 about here

\section{Survey Measures}


Table 3 shows the scales used to measure the constructs of this study: DTCA attitude, DTC attention, DTCA message perceptions, DTCA trust, general advertising beliefs (advertising in general), trust in mediated health information sources, trust in one's doctor, pharmaceutical corporate credibility, and behavioral intent to seek information from a doctor, the media, or interpersonal sources. All scales were based on established scales from prior literature as reported in the authors' previous publication (CITATION OMITTED FOR BLIND REVIEW) and were all measured on a Likert-type seven-point scale $(1=$ strongly disagree, $7=$ strongly agree $)$ unless otherwise noted). In addition to demographic characteristics, health-related characteristics were measured such as: self-rated health status (poor, fair, good, very good, excellent), number of doctor visits in the past six months, number of doctors visited in the past six months, and number of prescription medication taken in the last 6 months.

\footnotetext{
Place table 3 about here
}

\section{RESULTS}

\section{Preliminary Analysis}

The adequacy of variable-to-sample ratio and reliability and validity of the constructs were checked separately for the younger and older adults groups (as well as for the total sample). All constructs for both groups had an average variance extracted (AVE) scores above or equal to .53 and a construct reliability of above or equal to .84 , indicating good construct reliability and validity for both groups. A confirmatory factor analysis conducted for each group separately indicated a theoretically and statistically good measurement model fit (Younger Adults measurement model: $\chi^{2}=3409.68, d f=953, \mathrm{p}=.00 ; \mathrm{CFI}=.89 ; \mathrm{TLI}=.89 ; \mathrm{SRMR}=.05$ and Older Adults measurement model: $\left.\chi^{2}=2511.71, d f=953, \mathrm{p}=.00 ; \mathrm{CFI}=.93 ; \mathrm{TLI}=.92 ; \mathrm{SRMR}=.04\right)$. Factor loadings, AVEs and CRs can 
be seen in Table 3. The measurement model for both groups also had acceptable normed chi-square values (Younger Adults: $\chi^{2} / d f=3.57$; Older Adults: $\chi^{2} / d f=2.63$ ) with no correlations between constructs reaching .85. Acceptable values for the Fornell-Larcker criterion $\left[\mathrm{AVE}>(\mathrm{r})^{2}\right]$ confirmed discriminant validity, and multicolinearity was not evident ( $\mathrm{VIF}<5$ and tolerance $>.22)$.

Correlations among constructs can be seen in Table 4 along with descriptive statistics for each construct. All constructs were compared among younger and older adults with ANOVAs on SPSS, as a preliminary analysis, before running a multigroup SEM analysis using Mplus. ANOVAs could not be conducted for DTCA attitudes and trust in one's doctor due to the violation of the Levene's test. Significant differences were found among younger and older adults only for DTCA message perceptions $\left(F_{(1,1021)}=4.05, p<.05\right)$, DTCA trust $\left(F_{(1,1034)}=14.55, p<.01\right)$ and behavioral intentions $\left(F_{(1,1034)}=9.29, p<.01\right)$.

Place table 4 about here

\section{Differences between Younger and Older Adults}

A multigroup structural equation modeling analysis was conducted on the total sample with age as the grouping variable (younger adults versus older adults). To examine age differences, (a) an SEM model where all parameters (coefficients) between variables were allowed to vary by age group, and (b) an SEM model where all parameters (coefficients) were constrained to be equal between the two groups were compared (while holding the same measurement model). A chi-square difference test was then employed to test for significant differences between these two models ( $a$ and $b$ ) to verify whether differences between age groups exist for the theoretical model.

The analysis indicated significant differences between the (a) free parameters and (b) fully constrained models $\left(\Delta \chi^{2}=6188.36-6138.05=50.31, d f=2006-1980=26, \quad p<.01\right)$, implying significant 
differences exist between the two age groups. The free parameters SEM model indicated a statistically acceptable model fit across the two age groups $\left(\chi^{2}=6138.05, d f=1980, \mathrm{p}=.00 ; \mathrm{CFI}=.91 ; \mathrm{TLI}=.90\right.$; $\mathrm{SRMR}=.05)$. However, the model fitted the data for the older group $\left(\chi^{2}=2615.11\right)$ better than the younger group $\left(\chi^{2}=3522.94\right)$, illustrating that the model might be more relevant for older than younger adults in terms of predicting behavioral intentions. Results of the analysis can be seen in Table 5 as well as Figures 2 and 3.

Place table 5 and figures 2 and 3 about here

There were five relationships that differed between the younger sample and older sample SEM results. (1) Advertising in general significantly increased DTCA attitudes $(\beta=.12, p<.01)$ for the younger group, while this relationship remained non-significant among the older group. (2) DTCA message perceptions had a positive and significant relationship with DTCA attentions for the older group $(\beta=.36, p<.01)$, while this was not significant for younger group. (3) The greater the pharmaceutical corporate credibility of the older group the less attention was paid to DTCA ( $\beta$ $=.16, p<.05$ ), while this relationship was not significant for the younger group. (4) Also, older consumers who trusted their doctor more were significantly less likely to have favorable DTCA attitudes $(\beta=-.08, p<.05)$, while no significant relationships existed between these two constructs for the younger group. Lastly, trust in mediated health information sources had positive and significant relationships with (5) DTCA attention $\beta=.18, p<.01)$ and (6) behavioral intent $(\beta=.11$, $p<.01)$ for the older group, while these relationships were not significant for the younger group.

\section{Post-hoc Analysis}

Since health quality varies with age, further analysis examining the interaction of health status and age was conducted to determine the influence of age on model relationships when accounting for health. An overall health index was created by first standardizing each of four health 
characteristics (self-rated health status, number of doctor visits, number of doctors visited, and number of prescription medications) and then calculating an average index score across the four standardized measures. This overall health standardized index was divided by a median spit and dummy coded for lower health and better health groups (lower health [-1]: below -.134; better health [1]: -.134 and above) that were multiplied by dummy coded age groups (younger [1] and older [2] adults) to create four distinct groups: (1) younger adults and lower health, (2) younger adults and better health, (3) older adults and lower health, and (4) older adults and better health.

A one-way ANOVA comparing mean scores for each of the model variables between the four age and health groups revealed several significant differences (see Table 6). Notably, attention to DTCA differed by health status regardless of age, evaluations of the ads trended lower among older adults, trust in ones' doctor was highest among the older group with lower health, and behavioral intentions were highest among the younger group with lower health.

Place table 6 about here

A multigroup SEM model across the aforementioned four groups indicated significant differences between the fully constrained and free parameters models $\left(\Delta \chi^{2}=9100.38-8995.40=104.98, d f=4112\right.$ 1034=78, $p<.05$ ), implying significant differences exist between the four groups (see Table 7 and in Figures 4 through 7). The free parameters model indicated a statistically acceptable model fit across the four groups $\left(\chi^{2}=8995.40, d f=4034, p=.00 ; \mathrm{CFI}=.89 ; \mathrm{TLI}=.89 ; \mathrm{SRMR}=.06\right)$. The older adults and better health group had the lowest chi-square value $\left(\chi^{2}=1758.57\right)$, indicating that the data fit this group better than the other three groups. However, the independent variables accounted for the most variance in behavioral intentions (70.5\%) among the younger and lower health group and the least $(60.3 \%)$ among the older and better health group. 
Place table 7 and figures 4 through 7 about here

Some of the major differences between these four groups were: (1) DTCA trust did not have a significant relationship with DTCA attention for the two lower health groups, whether younger or older adults. (2) DTCA attitudes did not significantly impact behavioral intentions for the younger and lower health group. (3) Except for the effects of DTCA message perceptions and pharmaceutical corporate credibility, which positively and significantly impact DTCA trust across the four groups, the effects of advertising in general, trust in mediated sources, and trust in one's doctor on DTCA trust differed significantly among groups. (4) In turn, DTCA trust impacted negatively and significantly behavioral intentions only for the older groups (both lower and better health).

\section{DISCUSSION}

While previous descriptive work in the DTCA literature has produced inconsistent findings regarding the presence of age differences, the results presented here support the important role age plays in modifying the factors that are relevant to prescription drug ad responses. The pattern of variable relationships for the younger group was fairly straightforward and reflective of traditional advertising effects. Trust in DTCA was primarily based on a combination of message and source credibility perceptions with more minor contributions from general attitudes toward advertising and trust in mediated health information sources. In turn, greater trust and more positive attitudes toward advertising in general were associated with increased attention to the ads, and increased attention, along with positive attitudes toward DTCA and favorable perceptions of message accuracy and value, enhanced the likelihood of engaging in a behavioral response to the ads. 
By comparison, older adults exhibited a far more complex web of factors predicting responses to DTCA. While DTCA trust had completely mediated the effects of message perceptions, perceived pharmaceutical corporate credibility, and trust in mediated health information sources on attention among younger adults, these variables had additional direct relationships with attention among older adults. Older adults' behavioral intentions also had additional significant predictors in DTCA trust and trust in mediated health information sources along with the message perceptions, attention, and DTCA attitudes that had also been factors for younger adults. In addition, trust in one's doctor had been largely irrelevant for young adults but had significant, albeit small, inverse relationships with DTCA trust and DTCA attitudes among older adults.

Altogether, the wider set of factors associated with each of the DTCA ad outcomes for older adults could signify they are more discerning than younger adults in their decisions to devote attention to prescription drug ads and seek additional information following ad exposure. Such careful consideration is consistent with the greater risk averseness associated with seniors (Simcock, Sudbury, \& Wright, 2006) and their application of contextual knowledge and a broader set of cues to facilitate information processing and decision-making (Brown \& Park, 2003). In particular, in accordance with the assertions of socioemotional selectivity theory, trustrelated elements (DTCA trust, perceived pharmaceutical corporate credibility, and trust in mediated health information sources) were more predictive of a wider set of ad outcomes for the older adults in our study compared to the younger adults.

Our results also echo previous studies indicating that older adults' deference to a healthcare provider conflicts with positive regard for prescription drug ads (Menon, Deshpande, Perri, \& Zinkhan, 2002). Past research indicates a perspective among older adults (not 
necessarily shared by younger cohorts) that trust, in one's doctor for example, negates the need to ask further questions (Morris, Grossman, Barkdoll, \& Gordon, 1987). Thus, older adults' respect for authority may preclude the need to rely on information from prescription drug ads and study these ads carefully, resulting in reduced evaluations of this ad category. This may explain the inverse relationships we found between DTCA trust and behavioral intentions and between perceived pharmaceutical corporate credibility and attention for older adults as well. Perceiving pharmaceutical companies as credible could mean seniors see no need to carefully inspect the ads to learn further information about the drugs, and holding trust in DTCA could reduce seniors' inclination to check with a doctor, the media, or close others to verify ad information. The flipside is implied as well - older adults trust DTCA more if they do not have a trusting relationship with their doctor; attention to DTCA may be motivated in part by skepticism toward pharmaceutical companies; and seniors who talk to a doctor or seek other sources of information following ad exposure are more likely to be assessing the validity of ad claims.

Considering the model as a whole, the more varied interrelationships we found among variables for older adults may be a poignant depiction of seniors' ambivalence toward DTCA. Previous qualitative research has been especially insightful in capturing older adults’ appreciation of DTCA for raising awareness of prescription drug options but dissatisfaction with the specificity of ad information and overall lack of trust in the ads (DeLorme, Huh, and Reid, 2007; Grenard et al., 2011). Our findings perhaps add quantitative substantiation to this portrayal of older adults holding mixed views of DTCA and mixed motivations for responding to the ads. In contrast, younger adults appear to be much less conflicted.

The consideration of health status reveals further variation within age groups. Among older adults, it is those with better health who show higher attention to DTCA if they perceive 
pharmaceutical corporations to lack credibility, have lower attitudes toward DTCA if trust in their doctor is higher, link their overall trust in DTCA to attention and attitudes toward the ads, and exhibit an expanded role for trust in mediated health information sources in relation to attention and behavioral intent. In addition, their attitudes toward advertising in general are less of a factor in DTCA trust and attention. Altogether, this may indicate that seniors with fewer health issues are more likely to dissociate DTCA from other forms of advertising and are more selective in their response to DTCA. Older adults with more health concerns may instead be more receptive of DTCA as a supplementary source of health information addressing their health needs and have less weariness toward pharmaceutical corporations given the greater number of drugs they are taking.

It is also intriguing that younger adults with lower health have the fewest factors predicting DTCA attitudes and trustworthiness, and these evaluations had no significant bearing on the amount of attention paid to the ads nor their behavioral intentions. Instead, attention and specific perceptions of message value and accuracy were the sole drivers of behavioral intent. It is notable that this group expressed significantly greater likelihood to seek additional information about advertised drugs than their young counterparts with better health and older adults. Given that younger individuals engage in more information-seeking in general (Mata \& Nunes, 2010), and the assertions of socioemotional selectivity theory that information-seeking is more goalcongruent for younger adults (Carstensen, Isaacowitz, \& Charles, 1999), it makes sense that seeking information about a prescription drug after seeing it advertised is a fairly natural response of younger adults with greater health issues. As such, this group would not feel the need to scrutinize ads and be quite as restrictive as older adults in following up on a prescription drug ad. 


\section{Implications}

Based on the results, there appears to be a divergence between older and younger in the role of DTCA trust in stimulating further response to DTCA. For older adults, prescription drug information-seeking following ad exposure may be seen as a means of reducing uncertainty and is used on a more limited basis. Younger adults instead may engage in more evaluation of ad claims on their own and approach their doctors not to verify the validity of ad claims about which they are skeptical but to pursue attaining a drug based on ad claims they believe. This fits previous findings that older adults are more likely to simply ask about an advertised drug but are less likely than younger adults to request particular drug brands (Choi \& Lee, 2007; Datti \& Carter, 2006; Hausman, 2008; Mehta \& Purvis, 2003). While previous studies have examined age differences in level and sources of drug information search (Herzenstein, Mizra, \& Posavac, 2004; Lee, Salmon, \& Paek, 2007), the motivational nature of that information search is a largely unexplored area of research. Likewise, it is important for healthcare providers to be aware that motivations for discussing advertised drugs may differ between older and younger patients. For example, if younger are more trusting of advertised claims, they may have more misconceptions that require correction.

In addition, advertisers should take heed that message accuracy and usefulness was the most important factor overall for all sample groups but particularly among the older adults comprising the primary target market for prescription drugs. While regulations compel a certain degree of comprehensiveness and accuracy of ad information, advertisers should pay close attention to the types and style of information that older adults find most useful, comprehensible, and easy to evaluate if they wish to enhance seniors' perceptions of DTCA message and reduce the distrust that appears to underlie their ad responses. 


\section{Limitations}

Conclusions derived from this analysis should of course be tempered by the inherent limitations of our study. Two broad age groupings do not adequately cover the "life-span approach" quite as Southwell (2010) is recommending. For instance, we combined those in their late teens and early twenties with those in their mid-late forties, but previous research has shown college age individuals have a slightly different orientation toward DTCA than middle age adults (Ball, Manika, \& Stout, 2011). On the other end of the age spectrum, those who qualify as the "old old" are likely to face more health challenges, greater cognitive deficits, and have a shorter time horizon relevant to socioemotional selectivity theory compared to those who are newer members of the senior citizens group. Although our age distinction is similar to others (e.g., Huh, DeLorme, \& Reid 2004b; 2005), the definition of "old" can vary and thus should be noted when interpreting our results.

Dividing the sample into dichotomous groups as we did is admittedly a simplification that does not fully account for the full continuum of age and health status nor does it account for other factors identified by previous scholars (e.g., Menon et al., 2004) such as health involvement, channel characteristics, and ad execution elements that are likely to play a moderating role as well. Similarly, our approach addresses the aggregate level of DTCA response, but variations have been shown depending on the severity and chronicity of particular health conditions and treatments indicated in drug ads (Hausman, 2008). In addition, the results presented here are based on recalled exposure to DTCA, but evaluations may differ when exposure is immediate. Given the remarkably distinctive patterns of DTCA response we found for differing age and health status segments, further research compensating for the aforementioned limitations is warranted. 
References 